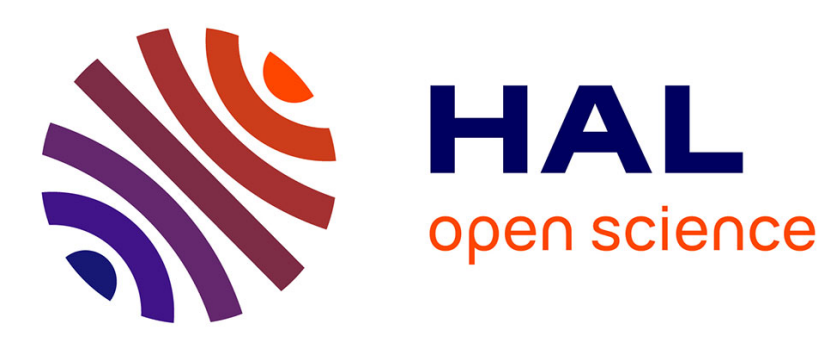

\title{
Un mode de justification en japonais: L'emploi de mono en fin d'énoncé
}

\author{
Jean Bazantay
}

\section{To cite this version:}

Jean Bazantay. Un mode de justification en japonais: L'emploi de mono en fin d'énoncé. Faits de langues, 2015, Varia, 45/2015. hal-02343381

\section{HAL Id: hal-02343381 \\ https://hal-inalco.archives-ouvertes.fr/hal-02343381}

Submitted on 2 Nov 2019

HAL is a multi-disciplinary open access archive for the deposit and dissemination of scientific research documents, whether they are published or not. The documents may come from teaching and research institutions in France or abroad, or from public or private research centers.
L'archive ouverte pluridisciplinaire $\mathbf{H A L}$, est destinée au dépôt et à la diffusion de documents scientifiques de niveau recherche, publiés ou non, émanant des établissements d'enseignement et de recherche français ou étrangers, des laboratoires publics ou privés. 


\title{
Un mode de justification en japonais : L'emploí de mono en fin d'énoncé
}

\author{
Jean Bazantay*
}

\section{INTRODUCTION}

Cet article traite de l'emploi du mot japonais mono en fin d'énoncé dans un échange interactif. Il a pour but d'éclairer ses valeurs énonciatives et son fonctionnement discursif pour nous interroger sur la pertinence de l'étiquette de «particule finale» (ou «énonciative») qu'on lui prête généralement dans cet emploi. Pour nous concentrer sur les valeurs spécifiques de ce terme, nous écarterons de notre champ d'investigation les distributions du type mono $\mathrm{ka}$, mono na, mono ne, etc. dans lesquelles mono apparaît en combinaison avec une particule finale. En revanche, nous intégrerons à la réflexion le mot mon que nous traiterons comme une variante familière du nom formel mono.

\section{EMPLOIS RÉFÉRENTIELS ET FONCTIONNELS DE MONO}

Le nom mono que les dictionnaires bilingues japonais-français traduisent par «chose», «objet» ou «article» est un des mots les plus fréquemment utilisés en japonais. Comme le mot français chose, il a la particularité de pouvoir tout aussi bien désigner un objet concret qu'un concept abstrait ou encore un ensemble de référents partageant les mêmes traits. Nom «caméléon» dont le référent change suivant l'environnement distributionnel ou le contexte d'énonciation, mono est aussi fréquemment employé à des fins purement fonctionnelles comme nom formel (keishiki meishi) ou nominalisateur propositionnel. Certains emplois fonctionnels s'accompagnent d'effets énonciatifs particuliers (expression de la surprise ou de la colère, injonction, etc.) que l'on peut relier à la dimension normative des énoncés. Les emplois référentiels et fonctionnels de mono ont fait l'objet de plusieurs descriptions en langues occidentales ${ }^{1}$ (Martin : 1975 ; Terada : 1992 ; Leboutet : 2003). L'analyse de la contribution sémantique du nom mono dans l'émergence de cette valeur déontique a aussi fait l'objet de plusieurs travaux (Aoki : 1994 ; Bazantay : 2012, 2013).

*INaLCO, CEJ-EA1441. Courriel : jean.bazantay@inalco.fr

1 Dans le cadre de recherches sur les keishiki meishi (noms formels) mono fait l'objet d'une littérature abondante en japonais. Voir notamment Teramura (1984), Morita (1989), Kitamura (2007, 2008, 2010), Momiyama (1991, 2000), Takahashi (2007, 2008), Iijima (2012). 
Comme dans la clôture de l'échange ci-dessous, mono est aussi fréquemment employé à la fin d'un énoncé syntaxiquement complet ${ }^{2}$.

(1)

$$
\begin{aligned}
& \text { saikin chikoku ga ooi yo! } \\
& \text { ces derniers temps retard-SUJ fréquent-PF } \\
& \text { Attention, tu es souvent en retard ces derniers temps! } \\
& \begin{array}{lll}
\text { datte sutoraiki de } & \text { densha ga } & \text { konai desu MONO. } \\
\text { mais-grève-CAUSE } & \text { train-SUJ } & \text { arriver-NEG COP-POLI MONO. } \\
\text { Que veux-tu que j'y fasse, } & \text { il n'y a pas de train avec la grève ! }
\end{array} \\
& \text { (Dictionnaire Shinmeikai) }
\end{aligned}
$$

D'un point de vue grammatical, mono ne semble pas remplir ici de fonction nominale ; sur le plan distributionnel, il se rapproche plutôt d'une particule finale $(\mathrm{PF})$ à laquelle il est parfois assimilé. Certaines grammaires ou dictionnaires (Meikyô, Daijirin) traitent d'ailleurs de cet emploi dans une entrée spécifique, laissant ainsi suggérer qu'il s'agit d'un lexème distinct du nom ${ }^{3}$.

Le fait qu'il s'agisse du même lexème ne fait toutefois aucun doute en raison de la proximité sémantique des deux emplois. On peut le vérifier en comparant la phrase ci-dessous avec l'exemple précédent.

$$
\begin{array}{lll}
\text { sutoraiki de } & \text { densha ga } & \text { konai MONO desu. } \\
\text { grève-CAUSE } & \text { train-SUJ } & \text { arriver-NEG MONO COP-POLI } \\
\text { Il n'y a pas de train avec la grève. } &
\end{array}
$$

En (2), mono est un nom formel ayant pour fonction de transformer une proposition (partie de gauche) en GN suivi de la copule assertive desu.

Les différentes présentations lexicographiques traduisent alors plutôt une différence de perception de l'autonomie de ces lexies entre elles et différentes conceptions des processus conduisant à leur utilisation en discours. Considéré dans une chaîne fonctionnelle comme l'aboutissement d'un processus de grammaticalisation, le mono conclusif de l'exemple (1) peut alors être traité comme un objet ayant acquis une autonomie et assimilé à une particule finale. En revanche, s'il est envisagé sous l'angle des théories énonciatives comme un constituant au service de la construction du sens, mono sera considéré comme une seule et même unité dotée de fonctionnements spécifiques et invariants suivant sa position dans la phrase.

Notre essaierons de montrer ici pourquoi cet angle énonciatif semble plus adapté pour comprendre cet emploi.

\footnotetext{
2 Voir liste des abréviations en fin d'article.

${ }^{3}$ De prime abord cette hypothèse pourra peut-être sembler incongrue aux japonisants. Néanmoins, dans le Koten kiso go jiten (Dictionnaire des mots fondamentaux de la langue classique), Ôno distingue deux noms distincts : $\mathrm{MONO}_{1}$ (物, 者) et $\mathrm{MONO}_{2}$ (鬼, 霊). Selon Ôno, le sens originel de $\mathrm{MONO}_{1}$ était «Ce que l'on ne peut changer, qui est immuable» alors que $\mathrm{MONO}_{2}$ désignait l'esprit malfaisant d'un mort (tama 霊) animé d'un fort ressentiment (urami 怨). (Ôno, 2012: 1247)
} 


\section{LES PARTICULES FINALES}

En japonais, les particules finales (ou énonciatives) sont des affixes qui apparaissent en fin de phrase après un énoncé syntaxiquement complet ${ }^{4}$. Elles expriment l'attitude communicationnelle du sujet parlant dans l'acte d'énonciation et sont donc essentiellement employées dans des interactions. Elles peuvent être de différentes natures : assertoriques $(s a, w a)$, interrogatives (ka, kai, ka na, kashira), confirmatives (ne, na), de mise en garde (yo, zo, ze), exclamatives (nâ,wa), interdictives (na), vérificatives (kke), etc.

Une particule finale n'est pas exclusive d'une autre et l'on observe fréquemment plusieurs particules finales l'une après l'autre suivant un ordre établi ${ }^{5}$. Cela montre qu'elles ne sont pas de même nature sur un plan énonciatif. Ainsi les particules liées à la transmission de l'information (assertion, interrogation, avertissement) précédent toujours les particules en rapport avec la relation interpersonnelle, ce qui corrobore les modélisations de la phrase japonaise (Masuoka : 2007; Nitta : 2003, 2009; Minami : 1995) en strates énonciatives suivant lesquelles, plus un élément se trouve à la périphérie de la phrase, plus son caractère subjectif est grand. Compte tenu de leur dimension énonciative, l'utilisation de ces particules présente des différences notables suivant le sexe, l'âge ou la région d'origine des locuteurs.

Comme les autres particules, les particules finales sont des mots non autonomes dépourvus de valeur référentielle. À celles-ci sont parfois assimilés certains mots (mono, koto, wake, etc.) par ailleurs utilisés comme noms dans des emplois référentiels ou fonctionnels.

\section{VALEUR DE MONO EN FIN D’ÉNONCÉ}

Selon le Nihon bunpô daijiten (Grand dictionnaire de la grammaire japonaise, 1971 : 846), la particule finale mono «s'emploie lorsque le locuteur explique les motifs de son comportement ou de sa pensée pour s'opposer à son interlocuteur, faire part de son mécontentement ou exprimer une revendication». L'ouvrage précise qu'elle est utilisée avec une nuance affective dénotant une certaine dépendance psychologique qualifiée d' «amaeta taido ${ }^{6}$ " (appel à la compréhension, demande d'indulgence, ton légèrement cajoleur). L'observation des contextes d'emploi montre par ailleurs que le locuteur est dans une position «défensive» dans laquelle il se sent contraint de s'expliquer ou de rétablir ce qu'il ressent comme une injustice. Dans le contexte d'une relation affectivement connotée, mono servirait ainsi à se justifier ou à réfuter une critique implicite

${ }^{4}$ Elles suivent en général une forme verbale ou la copule assertive à une forme conclusive. Dans des phrases nominales, elles apparaissent parfois après un nom lorsque la copule est omise.

${ }^{5}$ Pour un tableau détaillé des combinaisons, voir Nitta (2003:240).

${ }_{6}$ Ce concept renvoie à l'attitude psychologique connue sous le nom d'amae analysée par Doï Takeo dans Amae no kôzô (Traduction française : Le jeu de l'indulgence). 
par la présentation d'une information à valeur explicative (exposé de la raison, du motif, de l'arrière plan, etc.).

(3)

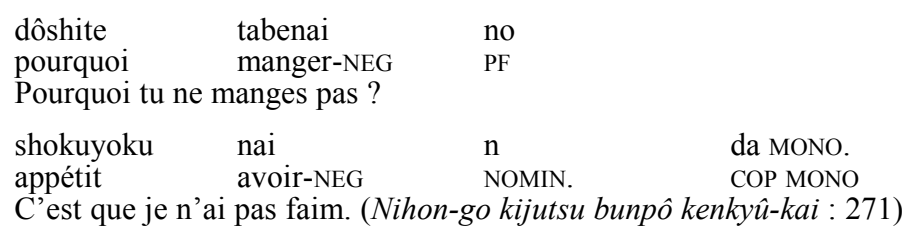

Si la valeur argumentative de mono fait consensus dans les grammaires, nous ne trouvons en revanche aucune explication permettant de la relier à certains traits du constituant mono. Nous aimerions ci-après proposer quelques hypothèses relatives au rôle de ce marqueur dans l'émergence de cet effet de sens.

\section{CONSTRUCTION DE LA VALEUR ARGUMENTATIVE}

Pour expliquer l'émergence d'une valeur argumentative d'ordre justificatif, notre hypothèse est que mono diffère des autres particules énonciatives purement fonctionnelles dans la mesure où il mobilise une partie de son caractère nominal, tant d'un point de vue référentiel que de celui des propriétés exclusives du substantif dans la phrase.

\subsection{Image référentielle du substantif mono (chose, objet)}

En convoquant en fin d'énoncé l'image matérielle et stable associée à son référent, la mention du concept de mono sur lequel le sujet n'a pas prise contribue à la construction d'une justification en présentant un phénomène extérieur qui s'impose et, en quelque sorte, dédouane le locuteur de toute responsabilité personnelle. Cela renforce les arguments présentés et «contrecarre» le reproche latent contenu dans le discours de l'interlocuteur en présentant les choses comme le résultat d'une norme générale ${ }^{7}$, d'une logique naturelle ancrée dans le sens commun.

Mais ce mono qui s'impose n'est pas un objet extérieur non identifié. Il est en relation avec la phrase qui le précède dont il pose le contenu propositionnel comme une entité incontournable, «que l'on ne peut changer» (Ono: id.). Examinons dans le paragraphe suivant comment cette opération se réalise.

\subsection{Image conceptuelle convoquée par le support nominal}

Nous faisons ici allusion à une propriété fondamentale des substantifs japonais, à savoir, leur capacité à régir d'autres éléments. Ce qu'illustre bien le

7 Tsubone (1996) utilise le terme de ippansei (caractère général) pour décrire cette valeur constante de mono. 
mécanisme dit de «nominalisation» par lequel un noyau nominal peut régir une proposition et transformer son contenu propositionnel en un concept nominal. C'est notamment l'une des fonctions du nom formel mono. D'un point de vue distributionnel, puisqu'en japonais l'élément déterminant est toujours antéposé à celui qu'il détermine, cet emploi de mono en fin d'énoncé présente certaines similitudes avec le schéma de la détermination nominale par une proposition.

Dans la langue contemporaine, du fait de la disparition progressive à l'époque d'Edo (1603-1867) d'une forme verbale adnominale spécifique dite rentai, la forme adnominale se confond désormais avec la forme shûshi (conclusive) n'ayant pas à l'origine vocation déterminante. En l'absence de pronom relatif en japonais, c'est alors cet ordre des mots qui permet de reconnaître une proposition déterminante. Même si dans la grammaire scolaire, seules les formes conclusives neutres sont considérées comme acceptables pour remplir cette fonction déterminante, en discours, de nombreux cas où le substantif est précédé d'une forme dite «polie» caractéristique d'un énoncé conclusif sont attestés. Vérifions-le par quelques exemples.

$$
\begin{aligned}
& \text { tsugi-ni mairimasu densha wa futsû himeji yuki } \\
& \text { après venir-POLI-train-THomnibus-Himeji-destination } \\
& \text { Le prochain train entrant en gare desservira toutes les gares jusqu'à Himeji. }
\end{aligned}
$$

En (4), le nom densha (train) est précédé de la proposition déterminante «tsugi ni mairimasu» («qui entre en gare»). Le verbe mairu (venir) se termine par le suffixe masu caractéristique d'une forme polie.

On rencontre également des syntagmes du type «tabemasu mono» («les aliments que je mange»), «ikimasu kuni» («le(s) pays où je vais») dans lesquels les noms mono (chose) et kuni (pays) sont respectivement précédés des verbes taberu (manger) et iku (aller) à une forme dite «conclusive polie». Même s'il s'agit de cas exceptionnels, l'absence de forme neutre devant le substantif ne semble donc pas suffisante pour écarter une relation de type déterminante.

De manière analogue, notre hypothèse est que l'émergence du mot mono à la fin de l'énoncé a pour effet d'opérer un recadrage conceptuel du procès vers un concept nominal. Même si, d'un strict point de vue syntaxique, la dimension nominale est discutable, on ne saurait nier que mono a conservé ici une partie de sa dimension conceptuelle de nom et qu'il modifie le sémantisme de la phrase en conférant à l'énoncé la stabilité et l'existence autonome propre aux références nominales. Dans cette lecture, l'énoncé se résumerait alors à un syntagme nominal dont la tête serait le noyau mono.

Ainsi, et indépendamment de certains traits sémantiques spécifiques de mono, cette occurrence nominale en fin d'énoncé concourt à la création d'une image conceptuelle qui participe à la construction de la réfutation ou de la justification.

\section{DATTE}


Dans l'échange, la collocation datte très fréquemment utilisée comme connecteur pour introduire la réponse joue un rôle important dans la construction argumentative. Observons son fonctionnement discursif à travers l'exemple ci-dessous :

(5)

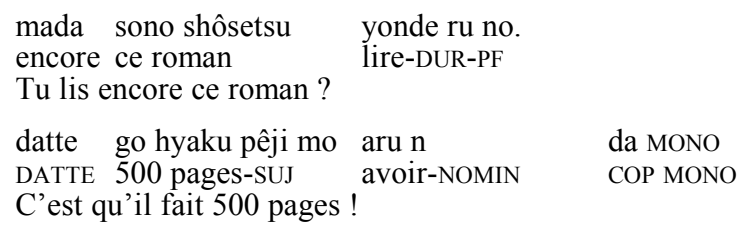

Datte est la forme contractée de «da to itte» (litt. : «même si tu dis cela»), expression dans laquelle on retrouve la valeur de citation de ce mot. Datte reprend ainsi le discours de l'interlocuteur («da to») avec une valeur adversative. Dans l'exemple qui nous intéresse, on peut le gloser par: «Tu me reproches de n'avoir pas encore terminé ce livre mais...». Le locuteur exprime implicitement que le discours de l'interlocuteur ne rend pas complètement compte de la réalité à laquelle il souhaite ajouter un élément objectif («le livre fait 500 pages»). En d'autres termes : «Ton reproche implicite n'est pas fondé car tu n'as pas pris en compte l'élément objectif (mono) que constitue la longueur de ce roman». Comme on le voit, dans cette valeur réfutative, datte permet de «rebondir» sur les propos de l'interlocuteur et d'introduire avec celui-ci une forme de confrontation.

On comprend alors que datte ne puisse s'employer dans un cadre social réclamant une certaine retenue et qu'il relève plutôt d'une situation langagière propice à l'expression spontanée des sentiments (amis, époux ou parents /enfants).

\section{DE L’ARGUMENTATION OBJECTIVE À LA MANIPULATION AFFECTIVE}

Datte peut renforcer le côté «amaeta» (complaisant, capricieux), notamment en cooccurrence avec «da mon» ou «desu mono». Comme nous l'avons vu au paragraphe 4, mono véhicule une nuance générique qui attribue aux circonstances exposées une force à laquelle on ne peut que se soumettre. Lorsque la raison invoquée appartient à la réalité objective, cela ne pose aucun problème et l'interlocuteur est prêt à l'accepter comme telle. Toutefois, lorsque les circonstances exposées ont une dimension subjective, cela confère un aspect arbitraire au propos dans la mesure où le locuteur présente comme incontournable, nécessaire, quelque chose qui est finalement du domaine subjectif. Il y a en quelque sorte un détournement de l'effet argumentatif de mono qui serait inacceptable dans une relation sociale dans laquelle chacun doit assumer la responsabilité de ses actes.

Entre adultes et enfants unis par un lien affectif, cela peut devenir une forme de jeu par lequel les adultes font preuve de complaisance face à une argumentation un peu spécieuse, à laquelle ils font semblant de croire. De son 
côté, le locuteur a également conscience de cette dimension et l'échange permet en quelque sorte de confirmer une relation affective de dépendance. La nuance d'amae «indulgence» peut être interprétée dans cette logique qui ne saurait avoir cours dans une relation entre adultes responsables.

L'exemple suivant est une illustration d'un tel emploi de mono car la réponse fournie par l'enfant et présentée comme une réalité objective n'est en fait guère recevable.

$$
\begin{aligned}
& \text { dôshite sono omocha ga hoshii no ? } \\
& \text { Pourquoi veux-tu ce jeu? } \\
& \text { datte, minna motteru } \mathrm{n} \text { da MONO. } \\
& \text { DATTE tout le monde avoir-TE-DUR NOMIN COP MONO } \\
& \text { Tout le monde en a un! (Nihon-go kijutsu bunpô kenkyû-kai : 271) }
\end{aligned}
$$

Un jeu affectif similaire peut également s'observer dans le cadre d'une relation amoureuse dans laquelle la femme/maitresse/geisha joue un personnage capricieux ou boudeur en empruntant certaines tournures enfantines (notamment da mon). Le côté immature participe à la séduction et la relation de dépendance établie flatte en quelque sorte ici l'ego masculin. De part et d'autres, les enjeux dépassent ainsi le cadre de l'échange en lui-même.

\section{DE LA RÉFUTATION À L’EXASPÉRATION}

Avec un schéma intonatif particulier se traduisant en début de réponse par un allongement et une accentuation de la première syllabe de datte et une forte intonation montante en fin de phrase, la tournure «DAtte ... da $M O N »$ exprime aussi une certaine impatience ou l'exaspération de la part du locuteur qui doit se justifier, comme si les choses étaient pourtant évidentes ou que cela le fatiguait de devoir expliquer à nouveau quelque chose d'aussi criant à ses yeux. Même s'il est de plus en plus attesté chez des locuteurs de sexe masculin (Bazantay, 2013 : 327), cet emploi est considéré comme relevant plus spécifiquement de la langue féminine. Là encore, le sentiment d'exaspération révèle un comportement immature d'enfant gâté (amayakasareta kodomo) ou d'adolescente capricieuse qui n'est pas acceptable dans le cadre d'une situation sociale ordinaire.

La raison mise en avant peut ainsi être subjective et ne pas toujours relever du «sens commun». Hashimoto (1998) nomme cette logique qui n'existe parfois que dans l'esprit du locuteur, logique du locuteur (hanashite no ronri).

\section{EMPLOI DE MONO POUR SOUTENIR L'INTERLOCUTEUR}

Outre cet emploi «auto-justificatif», mono est parfois utilisé par l'interlocuteur pour corroborer, ou soutenir des dires du locuteur. Examinons l'échange suivant entre deux femmes de soixante ans : 
(7)
atashi ne
kondo ichinen
shujin inai kara,kekkô kimama deshô.
je-FILLER,
dorénavant un an
mari être-NEG car, libre COP-CONJ
pour moi,
yôri shinai
wa.
L'année prochaine, comme mon mari va être absent, je vais pouvoir faire ce dont j'ai envie. Je ne m'embêterai à faire la cuisine pour moi toute seule.
deshô ne. sore shô ga nai wa yo. mukashi kara chan
to
COP-CONJ-PF cela ne rien pouvoir y faire PF-PF. autrefois-depuis
bien

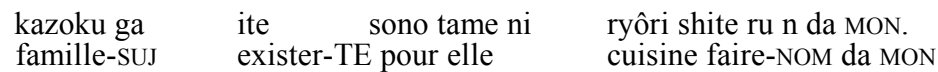
Naturellement. On va pas te le reprocher! Depuis le temps que tu fais la cuisine pour toute ta famille !

Selon les investigations de Wang (2007), cet emploi représenterait environ un tiers des occurrences de mono en fin d'énoncé dans des interactions. Par cet emploi, l'interlocuteur approuve explicitement les propos du locuteur en présentant lui-même un élément de justification ou de corroboration. Par le soutien qu'il apporte ainsi, cet emploi fonctionne comme un acte de langage permettant à l'interlocuteur de manifester une forme de solidarité, ici entre deux femmes ayant passé la majeure de partie de leur vie à s'occuper de leur famille. Il confirme un sentiment de proximité, d'appartenance au même groupe (miuchi, «familial»), notamment lorsque le locuteur est remis en cause par un tiers parti.

\section{DIMENSION CONNECTIVE DE MONO}

Si l'on envisage les échanges sous la forme de séquences explicatives, on constate que mono apparaît à la fin d'un énoncé $\left(\mathrm{E}_{2}\right)$ présentant un «phénomène expliquant» au sujet d'un «phénomène à expliquer» présenté précédemment dans la question ou la remise en cause $\left(E_{1}\right)$. $E_{1}$ et $E_{2}$ entretiennent donc une relation de type fait/justification (motif). Dans l'esprit du locuteur, le contenu propositionnel de $\mathrm{E}_{2}$ constitue une «bonne raison» qu'il relie au fait en question pour le justifier ${ }^{8}$. Cette opération de «validation» de $\mathrm{E}_{1}$ repose sur mono qui, en donnant au contenu propositionnel de $\mathrm{E}_{2}$ l'enveloppe conceptuelle d'une chose intangible, fonctionne comme un marqueur de la nécessité («Le fait incontournable est que...») ${ }^{9}$. Mono remplit donc ici une fonction de liage $\operatorname{argumentatif}\left(\mathrm{E}_{1}-\mathrm{E}_{2} \mathrm{MONO}\right)$.

8 Voir MacCready et Takahashi (2011) pour une proposition de formalisation élaborée.

9 Cet emploi argumentatif de mono n'est pas sans rappeler certaines utilisations du mot français chose qui a pour étymologie le nom latin causa désignant «ce qui est en discussion» (notamment devant un tribunal). Nous remercions le comité de rédaction d'avoir attiré notre attention sur ce point. 
Une relation explicative de même nature peut être observée avec la particule conjonctive no de qui permet de présenter des circonstances explicatives ou une raison objective.

(8) Sono hon wa takakatta NO DE kawanakatta. ce livre-TH cher-PASSÉ- NO DE acheter-NEG-PASSÉ Je n'ai pas acheté ce livre parce qu'il était cher.

La proximité de mono avec cette particule conjonctive peut être vérifiée dans la possibilité de substituer «mono de» à «no de». De la même manière, la plupart des échanges que nous avons envisagé jusqu'ici sous la forme ( $\left.\mathrm{E}_{1}-\mathrm{E}_{2} \mathrm{MONO}\right)$ peuvent être reformulés en une seule phrase sous la forme « $\mathrm{E}_{2} \mathrm{MONO} D E, \mathrm{E}_{1} »$.

Nous avons jusqu'ici envisagé des séquences explicatives du type fait/justification mais certaines similitudes peuvent également être observées avec l'explication causale. Examinons la phrase suivante dans laquelle apparaît kara, particule connective causale par excellence.
Kyô wa
musuko no tanjôbi
desu KARA,
hayaku
kaerimasu.
aujourd'hui-TH fils-de-anniversaire
COP-POLI-KARA
tôt

rentrer-POLI

Comme c'est l'anniversaire de mon fils aujourd'hui, je rentre tôt.

L'organisation discursive de cette phrase comprenant deux propositions $\mathrm{P}_{1}$ et $\mathrm{P}_{2}$ est :

$\begin{array}{ll}\mathrm{P}_{1} \text { kara (parce que) } & \rightarrow \mathrm{P}_{2} \\ \text { cause /raison } & \rightarrow \text { conséquence }\end{array}$

En regard de cette phrase, on observera la possibilité de l'échange suivant :

$\begin{array}{lll}\text { (10) dôshite } & \text { hayaku } & \text { kaerimasu ka. } \\ \text { Pourquoi } & \text { tôt } & \text { rentrer-POLI PF }\end{array}$

Pourquoi rentres-tu tôt?

kyô wa musuko no tanjôbi desu KARA.

aujourd'hui-TH fils-de-anniversaire-COP-POLI-KARA

Parce qu'aujourd'hui c'est l'anniversaire de mon fils.

En (10) kara qui clôt la réponse est en fait la particule connective de causalité observée en (9). La suite implicite de l'énoncé qui est contenue dans la question n'est pas répétée pour éviter la redondance. L'organisation discursive de cette séquence question/réponse présente ainsi certaines similitudes avec celle de l'exemple (1) reproche/ justification.

Mono s'inscrit donc dans le même paradigme que d'autres particules connectives à valeur explicative telles que kara, no de, sakai ${ }^{10}$, ken et son

${ }^{10}$ Ce mot appartient au dialecte d'Osaka. 
étiquetage courant de particule finale nous semble impropre compte tenu de la fonction conclusive caractéristique des particules finales.

$$
\begin{array}{l|l|l} 
& \begin{array}{l}
\text { da } \\
\text { desu/na } \\
\text { (ya) }
\end{array} & \begin{array}{l}
\text { kara } \\
\text { no de } \\
\text { mono, mon } \\
\text { sakai } \\
\text { (ken) }
\end{array} \\
\text { enfant } & \text { COP } & \begin{array}{l}
\text { Particule } \\
\text { explicative }
\end{array}
\end{array}
$$

Comme c'est un enfant, ...

\section{EMPLOIS DANS LE CADRE D’UN MONOLOGUE}

Nous nous sommes concentré ici sur des échanges du type «question-réponse» mais cette explication vaut également pour des propos tenus par une seule et même personne (au fil d'un récit, monologue, etc.).

Le fonctionnement de mono/mon en fin d'énoncé est le même. Son emploi traduit le sentiment de nécessité d'une forme de justification ou d'explication de la part locuteur. Il ne s'agit plus forcément de la justification d'un comportement précis, mais plutôt d'éléments de son récit. Observons l'exemple ci-dessous :

(11) Toshi-chan ni sonna koto ga zettai dekinai wa yo.
Toshi-DAT une telle chose-SUJ du tout pouvoir-NEG PF-PF Anata kenka nanka shinai MON. Toshi-chan wa itsumo ukenagashi da MON. tu querelle par ex. faire-NEG. Toshi-TH toujours esquive-COP-MON

$\mathrm{Tu}$ (Toshi-chan) es tout à fait incapable de faire quelque chose comme cela. Tu ne te fâches jamais. Tu laisses toujours tout glisser sur toi. (Kirino Natsuo)

En (11), mon apparaît à la fin de la seconde phrase comme support de l'explication de ce qui est avancé dans la première. On le retrouve une seconde fois dans la combinaison «da mon» à la fin de la troisième phrase. Il sert ici à fournir une explication sur la deuxième phrase, en l'occurrence il s'agit d'une explication de la raison pour laquelle elle ne se fâche jamais : «Tu laisses tout glisser sur toi.»

En servant de support à des explications successives, mon permet ainsi des «arrêts» sur certaines explications. Cet emploi rappelle celui de wake (raison) comme filler pour souligner légèrement une information.

\section{CONCLUSION}

Nous avons analysé les mécanismes sémantiques et pragmatiques sous-tendant l'émergence de la valeur de justification en la reliant à certaines 
propriétés référentielles ou grammaticales du nom mono. En visant à contrecarrer la pertinence du discours de l'interlocuteur, nous avons notamment montré comment l'objectivisation d'un élément explicatif réalisée par le truchement mono permettait de construire une opération de type argumentatif.

L'analyse discursive de quelques emplois a également permis de mettre en évidence la dimension elliptique des énoncés se terminant par mono, ce qui pose la question de la pertinence de son étiquetage courant de «particule finale». Dans cet emploi, nous avons également signalé une proximité distributionnelle et sémantique avec d'autres mots explicatifs tels que wake ou kara. Ces termes s'inscrivent en effet dans un même paradigme devant la copule assertive ou en fin d'énoncé dans une valeur connective.

Type «nominalisateur»

Type «connecteur»

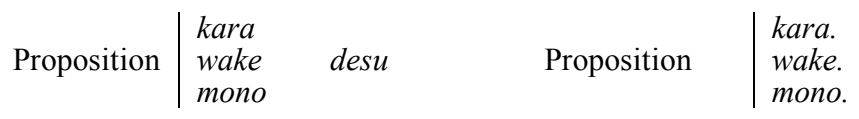

Outre ce rapport paradigmatique, mono et kara entretiennent également une relation d'ordre syntagmatique (... mono kara desu / mono da kara, etc.). Il serait donc intéressant d'approfondir la question des points de convergence et de divergence entre ces deux termes à valeur explicative.

\section{LISTE DE ABRÉVIATIONS}

$\begin{array}{ll}\text { CONJ } & \text { aspect conjectural } \\ \text { COP } & \text { copule assertive } \\ \text { DAT } & \text { marqueur du datif } \\ \text { DUR } & \text { aspect duratif } \\ \text { GN } & \text { groupe nominal } \\ \text { NEG } & \text { négation } \\ \text { NOMIN. } & \text { nominalisateur } \\ \text { PF } & \text { particule finale } \\ \text { POLI } & \text { style poli } \\ \text { SUJ } & \text { marqueur du sujet } \\ \text { TE } & \text { particule connective «te » } \\ \text { TH } & \text { marqueur du thème }\end{array}$

\section{BIBLIOGRAPHIE}

Aoki S, 1994, Nom de chose, détermination et énonciation : à propos du nom formel mono en Japonais, Travaux de Linguistique contrastive Franco-japonaise, Tokyo, Nichifutsugo taishô kenkyûkai, p. 134-148.

Bazantay J., 2012, A propos de mono en japonais: considérations sémantico-référentielles dans une approche contrastive avec le mot chose, Scolia 26, p. 39-60. 
Bazantay J., 2013, La chose pour le dire : mono en japonais contemporain: approche sémantique, syntaxique et énonciative, Thèse de doctorat, Université Bordeaux 3, Michel de Montaigne.

Doi T., 1988, Le jeu de l'indulgence: étude de psychologie fondée sur le concept japonais d'amae. Paris, L'Asiathèque.

Hashimoto Y., 1997, Shuujoshi mono: 'hanashite-no ronri'-kara-no setsumei (sentence final particle mono: Explanation under 'speaker's logic'), Studies on Japanese and Japanese Culture 7, Osaka University of Foreign Studies, p. 201-212.

Ijima M., 2012, mono da, koto da, wake da bun no kôzô to kinô (Structure et fonctions des phrases en mono da, koto da et wake da), Nihongogaku ronsh $\hat{u}$ 8, Université de Tokyo, p. 95-145.

Kitamura M., 2007, Mono da bun ni okeru jutsugo meishi mono no yakuwari (Rôle du nom prédicatif mono dans les phrases en mono da), Nihongo no kôzô henka to bunpô-ka (Changements structurels du japonais et grammaticalisation), Tokyo, Hitsuji shobô, p. 221-242.

Kitamura M., 2008, Odoroki, kangai o arawasu mono da bun no kôzô henka kinsei ikô o chûshin ni (Changements structurels des phrases en mono da exprimant la surprise ou les sentiments - à partir de l'époque moderne), Kokubun gaku 92 (Littérature japonaise 92), Osaka, Kansai University, p. 1-17.

Kitamura M., 2010, A pragmatic analysis of 'mono da' in Japanese, Journal of Nagoya Gakuin University Humanities and natural sciences 47 (1), Nagoya Gakuin University, p. 47-60.

Leboutet L., 2003, Noms et nominalisateurs: étude de koto, mono, no en japonais écrit contemporain, Thèse de doctorat l'EHESS, Paris.

Martin S., 1975, A reference grammar of Japanese, New Haven London, Yale University press.

Masuoka T., 2007, Nihongo modariti tankŷ̂ (Exploration de la modalité en japonais), Tokyo, Kuroshio Shuppan.

McCready E. \& Takahashi Y., 2011, Good reasons. Beyond Expressives: Explorations in Use-conditional Meaning, H.-M. Gärtner and D. Gutzmann, eds. Brill, p. 201-229.

Minami F., 1993, Gendai Nihongo bunpo no rinkaku (Contours de la grammaire japonaise contemporaine), Tokyo, Librairie Taishûkan.

Morita Y., 1989, Kiso Nihongo jiten (Dictionnaire du japonais fondamental), Tokyo, Kadokawa Shoten.

Momiyama Y., 1991, Modern Japanese meanings and uses of mono as used without modifiers, Studies in language and culture 13 (1), Université de Nagoya, p. 105-118.

Momiyama Y., 2000, Meishi 'mono' no tagi kôzô (Structure pluri-sémantique du nom mono), Nihon-go imi to bunpô no fûkei (Paysages sémantiques et grammaticaux du japonais), Tokyo, Hitsuji shobô, p. 177-191.

Nihongo Kijutsu Bunpô Kenkyûkai, 2003, Gendai Nihongo bunpô - 4 - modariti (Grammaire du japonais contemporain - 4- Modalités), Tokyo, Kuroshio Shuppan.

Nitta Y., 2009, Nihongo no modariti to sono shûhen (Les modalités en japonais et leurs contours), Tokyo, Hitsuji Shobô.

Takahashi Y., 2007, On studies on mono da: From a viewpoint of adnominal modifying constructions, Bulletin of the Foreign Student Education Center Tôkai University 27, Tokyo, Tôkaï University, p. 1-20. 
Takahashi Y., 2008, On the mono da Sentence with the content clause construction, Bulletin of the Foreign Student Education Center Tôkai University 28, Tokyo, Tôkaï University, p.17-36.

Terada A., 1992, Autour du nominalisateur mono, Recherche en linguistique japonaise Volume 3 la nominalisation, Université Paris VII, p. 121-137.

Teramura H., 1984, Nihongo shintakusu to imi II (Syntaxe et sémantique du japonais II), Tokyo, Kuroshio Shuppan.

Tsubone Y., 1996, Shû-joshi, setsuzoku joshi to shite no 'mono' no imi -'mono', 'mono nara', 'mono no', 'mono o' (Signification de mono en tant que particule finale ou particule conjonctive : mono nara, mono no, mono o), Nihongo kyôiku 91 (Journal of Japanese language teaching 91), Tokyo, Nihongo kyôiku gakkai, p. 37-48.

Wang Y., 2007, A functional study of the final particle mono in japanese conversational discourse. The linguistics journal Vol.2-1. Road Town (British Virgin Islands), Linguistics journal press, p. 162-183.

\section{Dictionnaires}

Daijirin (Grande forêt des mots), 2006, $3^{\mathrm{e}}$ édition, sous la direction de A. Matsumura, Tokyo, Sanseidô.

Koten kisogo jiten (Dictionnaire des mots fondamentaux de la langue classique), 2011, sous la direction de S. Ohno, Tokyo, Kadokawa Gakugei Shuppan.

Meikyô kokugo jiten (Dictionnaire de japonais du miroir clair), 2002, 1 ère édition, sous la direction de Y. Kitahara, Tokyo, Librairie Taishûkan.

Nihon bunpô daijiten (Grand dictionnaire de la grammaire japonaise), 1953, Sous la direction de A. Matsumura, Tokyo, Meiji Shoin.

Nihongo bunkei ziten (Dictionnaire des structures du japonais), 1998, sous la direction de Y. Sunakawa \& A. Bekes, Tokyo, Kurosio Syuppan.

Shin meikai kokugo jiten (Nouveau dictionnaire de japonais aux explications claires), 1997, 5e édition, sous la direction de K. Kandaïchi, Tokyo, Sanseidô. 